\title{
INVESTIGAÇÃO DA TEMPORALIDADE DAS CARACTERISTICAS DEFINIDORAS DO DIAGNÓSTICO DE RESPOSTA DISFUNCIONAL AO DESMAME VENTILATÓRIO
}

\author{
Investigation of the temporality of the defining characteristics of the diagnostic response \\ to dysfunctional ventilatory weaning \\ La investigación de la temporalidad de las características definitorias de diagnóstico de \\ la respuesta disfuncional para el destete ventilatorio
}

Théia Maria Forny Wanderley Castellões ${ }^{4}$

\section{RESUMO}

Estudo que investigou a distribuição temporal das características definidoras da Resposta Disfuncional ao Desmame Ventilatório. Teve como objetivos: descrever a conformação da distribuição cronológica das características definidoras desse diagnóstico em pacientes sob ventilação prolongada na fase de interrupção da ventilação mecânica e verificar a existência de um padrão temporal predominante. Trata-se de um estudo do tipo descritivo-exploratório com abordagem quantitativa. Foram sujeitos os clientes adultos internados por causas clínicas no Centro de Terapia Intensiva do Hospital Pró-Cardíaco, sob ventilação mecânica prolongada e que estavam em desmame. Com os resultados se obteve a confirmação da hipótese de existência de uma distribuição temporal predominante na amostra observada. Foi possível distinguir os principais intervalos de observação/manifestação das Características Definidoras, a saber: precoce, intermediário e tardio. Em relação ao gerenciamento do processo de desmame foi possível indicar algumas contribuições ao aprimoramento das avaliações protocolares.

Palavras-chave: Diagnóstico de enfermagem. Desmame do respirador mecânico. Distribuição temporal.

\begin{abstract}
This study investigated the temporal distribution of the defining characteristics Response to Dysfunctional Ventilatory Weaning. Aimed to describe the conformation of the chronological distribution of the defining characteristics of this diagnostic in ventilated patients during the prolonged interruption of mechanical ventilation and verify the existence of a prevailing weather pattern. This is a descriptive study and exploratory quantitative approach. The subjects were adult clients admitted for clinical reasons in the Intensive Care Unit, Hospital Pro-Cardiac under prolonged mechanical ventilation and who were being weaned. With the results was obtained to confirm the hypothesis of a temporal distribution prevailing in the observed sample. It was possible to distinguish the main intervals of observation I demonstration of the defining characteristics, namely: early, intermediate and late. Regarding the management of the weaning process could be given some contributions to better assessments of protocol.
\end{abstract}

Keywords: Nursing diagnosis. Ventilator Weaning. Temporal distribution.

\section{Resumen}

Un estudio investigó la distribución temporal de las características definitorias de Respuesta Disfuncional al Destete Ventilatorio. Tuvo como objetivo describir la conformación de la distribución cronológica de las características definitorias de este diagnóstico en pacientes sometidos a ventilación prolongada durante el destete de la ventilación mecánica y para verificar la existencia de un patrón climático predominante. Se trata de un estudio descriptivo de enfoque cuantitativo y exploratorio. Los sujetos fueron clientes adultos ingresados por razones clínicas en la Unidad de Cuidados Intensivos del Hospital Pro-cardiaco, bajo ventilación mecánica prolongada y que habían de ser destetados. Con los resultados se obtuvo la confirmación de la hipótesis de una distribución temporal que prevalecen en la muestra observada. Fue posible distinguir los intervalos principales de observación y de demostración de las características que definen, a saber: temprana, intermedia y tardía. En cuanto a la gestión del proceso de destete, se podría dar algunos aportes para una mejor evaluación del protocolo.

Palabras clave: Diagnóstico de enfermería. Desconección del Ventilador. Distribución Temporal

\footnotetext{
'Mestre em Enfermagem pela EEAN/UFR. Enfermeira Chefe do Centro de Terapia Intensiva do Hospital Federal da Lagoa. Membro do Grupo de Estudos em Aprendizagem e Cognição (GEAC). Pesquisadora do Grupo de Pesquisa Tecnologias e Concepções para a Sistematização da Assistência de Enfermagem (TECCONSAE). Rio de Janeiro - RJ. Brasil. E-mail: enfavellar@gmail.com; 2Doutor em Enfermagem. Professor Adjunto do Departamento de Enfermagem Fundamental da Escola de Enfermagem Anna Nery/UFR. Pesquisador Permanente do Grupo de Estudos em Aprendizagem e Cognição (GEAC). Líder do Grupo de Pesquisa Tecnologias e Concepções para a Sistematização da Assistência de Enfermagem (TECCONSAE). Rio de Janeiro - RJ. Brasil. Email: marcosbrandao@ufri.br; ${ }^{3}$ Doutora em Enfermagem pela UFRJ. Enfermeira do IFF-FIOCRUZ. Coordenadora do Curso de Pós-Graduação latu sensu “ Evolução do Cuidado de Enfermagem em Cardiologia baseado em Inovações Científicas e Tecnológicas - PROCEP/ Pró-Cardíaco. Rio de Janeiro R. Brasil. Email: Valzadra@uol.com.br; ${ }^{4}$ Mestre em Enfermagem pela UER. Enfermeira do Hospital Procardíaco Pronto Socorro Cardiológico S/A. Enfermeira Responsável pela Assistência Ventilatória no Hospital Procardíaco. Rio de Janeiro - R. Brasil. E-mail: theiacastelloes@gmail.com
} 


\section{INTRODUÇÃO}

A expressão diagnóstico de enfermagem (DE) tem duas aplicações principais: o de uma estrutura padronizada para a prática da enfermagem por meio do processo de enfermagem; e a de respostas aos problemas de saúde do cliente, em uma nomenclatura unificada (taxonomia).

No primeiro contexto, o diagnóstico está no processo de enfermagem e nele interfere norteando quais serão os passos seguintes, e de modo mais imediato, definindo as intervenções de enfermagem. Portanto, é pelo diagnóstico que se constrói um cuidado direcionado, especializado e específico a um cliente, com a visão do indivíduo e não apenas da sua condição física. Brandão 0:29 indica que "prescrever sem ter antes diagnosticado gera um cuidado baseado na necessidade usual de uma categoria de clientes que vivem uma situação clínica semelhante", o que resultaria em um cuidado geral, muitas vezes tarefeiro e não eficiente para as reais e particulares necessidades desse cliente único.

Considerando a relevância do diagnóstico de enfermagem é importante ter em mente o papel que os dados obtidos têm na definição de um apropriado julgamento clínico. Para que o enfermeiro possa construir o DE ele deve basearse em dados minuciosamente coletados, com informações precisas, que demonstrem com clareza as respostas aos "problemas" apresentados pelo cliente. A partir deste quadro de respostas podem-se gerar as hipóteses diagnósticas, já que os diagnósticos apresentam padrões característicos que servem como critério de definição.

No entanto, algumas dificuldades podem ser observadas neste processo de lidar com os dados. $\mathrm{Na}$ perspectiva da Enfermagem, existem quatro tipos de erros diagnósticos: 1) falhar na elaboração do diagnóstico, quando existem achados suficientes para indicar a presença do diagnóstico; 2) elaborar um diagnóstico, quando os achados não se afinam às características daquele diagnóstico; 3) fazer um diagnóstico geral, quando as evidências permitem um diagnóstico mais específico, preciso; e 4) fazer um diagnóstico específico, presumindo a existência de achados que de fato não estão presentes². Essas categorias de erros demonstram que a acurácia do diagnóstico está intimamente relacionada com a capacidade de avaliação do enfermeiro e com 0 conhecimento prévio dos diagnósticos de enfermagem.

Desta forma, alguns fatores são categorizados como interferentes na acurácia da decisão diagnóstica em: (a) relacionados à tarefa diagnóstica; (b) relacionados ao contexto situacional e (c) relacionados ao diagnosticador ${ }^{3}$. Tais categorias não incluem a precisão da descrição do diagnóstico, não levando em consideração o fato de que este pode não estar suficientemente descrito na taxonomia.

Assim, o presente estudo apresenta a hipótese original de que uma variável não descrita interfira na acurácia diagnóstica em enfermagem, sendo ela o padrão temporal das características definidoras do diagnóstico. Nesta situação, outra categoria de inacurácia em definir o diagnóstico teria origem na busca por evidências em circunstâncias temporais inapropriadas.

Neste contexto, sintetizam-se as possiveis falhas na descrição do padrão diagnóstico em três grandes tipos: 1) a definição do diagnóstico não é precisa ou clara, portanto, 0 enfermeiro não consegue compreender o que o diagnóstico abrange; 2) as características definidoras não são apropriadas ou suficientes, sendo necessários estudos de validação para reestruturar a descrição do padrão, incluir ou excluir características definidoras ou, até mesmo, invalidar o diagnóstico; e 3) as características definidoras não são objeto de investigação conforme a sua distribuição temporal, assim, 0 diagnosticador não consideraria qual(is) característica(s) definidora(s) deveriam anteceder as demais, o que poderia fazer com que ele buscasse evidências em tempos inadequados.

Partindo da pressuposição da existência de erros do terceiro tipo e da validade da defesa argumentativa desta temporalidade na identificação das características definidoras, ainda faltam as evidências de pesquisa. 0 presente estudo busca trazer contribuição ao preenchimento desta lacuna de conhecimento que pode influenciar a decisão diagnóstica acurada. Para tal investigou-se a perspectiva temporal das características definidoras do diagnóstico de Resposta Disfuncional ao Desmame Ventilatório (RDDV).

Sabidamente uma ampla eleição de fenômenos diagnósticos poderia ser feita para investigar o tempo em relação às características definidoras; contudo, no estudo se elege 0 caso particular da RDDV devido a três motivos principais: (a) a possibilidade de recorte temporal mais preciso por conta dos eventos de desmame; (b) a experiência da autora em unidades de terapia intensiva com pacientes sob ventilação mecânica e em processo de desmame ventilatório, pela qual foi possível perceber a alta prevalência do diagnóstico em tela e; (c) a significativa relevância clínica da RDDV, cuja identificação precoce tem impacto direto na recuperação e no bem-estar do paciente.

Desta forma, o estudo teve como objetivos:

- Descrever a conformação da distribuição cronológica das características definidoras do diagnóstico de enfermagem Resposta Disfuncional ao Desmame Ventilatório em pacientes sob ventilação prolongada na fase de interrupção da ventilação mecânica;

- Verificar a existência de um padrão temporal predominante para as características definidoras da Resposta Disfuncional ao Desmame Ventilatório em pacientes sob ventilação prolongada na fase de interrupção da ventilação mecânica;

\section{MATERIAIS E MÉTODOS}

Trata-se de um estudo descritivo-exploratório com abordagem quantitativa, alinhando a necessidade de conhecer um fenômeno específico ainda restrito. Nele se faz necessária a descrição detalhada, em que o pesquisador 
"observa, descreve e documenta vários aspectos de um fenômeno"4.

No presente estudo, o padrão cronológico das características definidoras do diagnóstico de enfermagem uma vertente não descrita pelos estudos do tema - foi caracterizado para apresentar sua distribuição temporal. Também, foi explorada a existência de uma sequência predominante entre elas. A natureza exploratória foi a primeira abordagem ao problema da temporalidade no diagnóstico, analisando como o fenômeno se manifesta e os outros fatores com o qual se relaciona.

Os sujeitos foram os pacientes adultos (acima de 18 anos) internados por causas clínicas nos centros de terapia intensiva (CTI) 1 e 2 do Hospital Pró-Cardíaco, um hospital referência em medicina de alta complexidade da rede privada do município do Rio de Janeiro. A instituição possui uma história pautada na busca pela qualidade, com investimentos não apenas na estrutura física da instituição e em tecnologias duras, mas também em quantidade e qualidade profissional, com capacitação e valorização dos recursos humanos.

Foram sujeitos aqueles pacientes internados nos CTI que estavam sob ventilação mecânica invasiva por mais de 24 horas e que foram considerados aptos a iniciar o processo de desmame ventilatório de acordo com a equipe do hospital em questão. Eventos de retirada da prótese ventilatória que foram realizados na ausência da enfermeira responsável pela ventilação mecânica e da pesquisadora foram automaticamente excluídos da amostra.

A pesquisadora coletou dados nos dias úteis e em horários que coincidiam com o da enfermeira da assistência ventilatória (no período da tarde). Os dias de coleta não foram predeterminados. Desta forma, foi acompanhado o evento de todos os pacientes que estivessem passando pelo processo de retirada da prótese ventilatória (conforme decisão da equipe) no momento em que a pesquisadora e a enfermeira da assistência ventilatória estavam presentes simultaneamente.

A coleta de dados aconteceu na fase de interrupção da ventilação mecânica, iniciada na retirada da prótese ventilatória e finalizada com o retorno à ventilação invasiva ou não invasiva, seja por insucesso no desmame ventilatório ou por determinação do protocolo de desmame ventilatório institucional ou, ainda, em até 24 horas após a retirada da prótese, sendo considerada, nesse último caso, como sucesso do desmame. Vale ressaltar, que na instituição é adotado um protocolo de desmame ventilatório progressivo, com a retirada da prótese ventilatória dos pacientes considerados crônicos, acontecendo em períodos de duração pré-programados, com o retorno para a ventilação mecânica de maneira eletiva, mesmo quando não é diagnosticada pela equipe a Resposta Disfuncional ao Desmame Ventilatório. Por esse motivo, a coleta de dados era interrompida nesse momento.

0 processo de desmame caracteriza-se pela "transição da ventilação mecânica invasiva para a espontânea nos pacientes submetidos à ventilação mecânica por tempo superior a 24 horas" 5:5128. Assim, tem duração for temente variável de caso a caso, sofrendo interferência da sua própria dinâmica, requerendo avaliação especializada. Contudo, no processo de desmame a retirada de prótese ventilatória é um procedimento marcante e que pode representar um marcador temporal do processo. Pela prática clínica, é possível determinar de forma intensiva as reações do indivíduo nesta fase.

Diante do referido, optou-se por coletar os dados durante este intervalo de tempo, considerando a relevância da fase de interrupção da ventilação mecânica. Por outro lado, existiria uma complexidade de coletar os dados ao se pesquisar todo o processo de desmame de maneira prospectiva, sem que se obtivessem vantagens significativas no alcance de resultados.

Para obtenção dos dados foi utilizada a observação estruturada, que esteve focada nos sinais e sintomas característicos da RDDV conforme a descrição taxonômica da NANDA-I. Deste modo, nem todas as manifestações potenciais foram documentadas. Assim, na manifestação de uma dada característica definidora, a pesquisadora registrava o horário de sua manifestação em função do início do processo de desmame (tempo zero). Tal desenho foi sistemático e objetivo.

Como método de coleta de dados, a pesquisadora iniciava o processo por uma avaliação inicial (busca dos sinais e sintomas característicos da RDDV) em todos os sujeitos observados antes da interrupção da ventilação mecânica, para obter o parâmetro de normalidade do sujeito e detectar o exato momento de manifestação da característica definidora. Em casos de incerteza, o parecer da enfermeira da assistência ventilatória e/ou da enfermeira plantonista era solicitado para consenso.

Aamostrafoi composta por 28 eventos de pelo menos uma característica definidora de RDDV para um total de 38 situações de desmame. Isso representou todos os eventos de interrupção da ventilação mecânica nas datas e horários reservados para a coleta dedados.

Há que se destacar que os dados apresentados no estudo representam o número de eventos de retirada de prótese, e não o número de sujeitos. Cada evento de retirada da prótese ventilatória pôde ser utilizado como um fenômeno único a ser pesquisado e, portanto, o mesmo indivíduo pôde ser sujeito da pesquisa em diversos episódios diferentes de interrupção da ventilação mecânica.

Após a coleta dos dados, estes foram organizados em uma planilha eletrônica baseada em Excel for Windows ${ }^{\odot}$, produzindo um mapa de dados com as características definidoras. 0 softwareem questão permitiu a realização das operações de análise dos dados. Tal análise foi desenvolvida tendo por base a estatística descritiva.

Considerando que todas as 203 características definidoras verificadas nos 28 eventos representavam uma distribuição global que poderia indicar a tendência da RDDV na amostra, foi selecionado o conceito de quartil e intervalo interquartil para estabelecer os pontos de corte entre o que representaria as situações de aparecimento precoce, intermediário e tardio. 
0 primeiro quartil representando os primeiros $25 \%$ do tempo total da distribuição observado foi considerado como precoce. O intervalo interquartil que corresponde o período compreendido entre os percentis 25 e 75 foi considerado como intermediário. Por fim, acima do percentil 75 ou após o terceiro quartil definiu-se o período tardio. A característica definidora de manifestação mais tardia apareceu aos 205 minutos, sendo este o intervalo temporal total da distribuição. As medianas para cada característica definidora (CD) foi a medida utilizada para alocar a CD em um dos três intervalos.

No que concerne aos aspectos éticos, o estudo foi dirigido pela Resolução 196/96 do Conselho Nacional de Saúde que aponta as diretrizes enormas regulamentadoras de pesquisas envolvendo seres humanos. 0 projeto de pesquisa foi submetido ao Comitê de Ética em Pesquisa (CEP) do Hospital Pró-Cardíaco e aprovado em 09/12/2009 sob o número de registro 326. 0 CEP dispensou o investigador de aplicar o Termo de Consentimento Livre e Esclarecido, considerando a pesquisa de risco mínimo.

\section{RESULTADOS E DISCUSSÃO}

Obteve-se como material para análise um total de 38 interrupções da ventilação mecânica, observadas em 14 sujeitos. Desses 38 eventos, a Resposta Disfuncional ao Desmame Ventilatório foi verificada em 28 eventos, de acordo com a observação da pesquisadora.

No que concerne à idade, os sujeitos tinham idade compreendida entre 53 e 93 anos, com predominância de idosos. E em relação ao sexo, foi observada predominância do sexo masculino (57\%) em relação ao sexo feminino (43\%). Esse predomínio pelo sexo masculino repete a tendência nacional de internações, ainda que a supere na amostra. As estatísticas nacionais no que diz respeito às internações de idosos (acima de 60 anos) obtidas do DATASUS ${ }^{6}$ referentes a agosto de 2010 indicam que $52 \%$ das internações foram de pacientes do sexo masculino.

Entre os diagnósticos médicos mais frequentes como causa da internação estavam a Doença Pulmonar Obstrutiva Crônica Descompensada (presente em 5 sujeitos) e a Sepse Pulmonar (presente em 4 sujeitos), o que corrobora o estudo de Constantino 7 realizado no Hospital Universitário Clementino Fraga Filho (HUCFF), que também aponta esses diagnósticos como as principais causas de necessidade de Ventilação Mecânica na Unidade de Terapia Intensiva.

Ainda estiveram presentes outros diagnósticos médicos com foco extrapulmonar, que de acordo com a gravidade e grau de comprometimento do paciente também podem ocasionar a necessidade de VM, como o Acidente Vascular Encefálico (AVE), a Fibrilação Atrial (FA) e o Infarto Agudo do Miocárdio (IAM).

Também foram elencadas as comorbidades presentes nos pacientes sujeitos da pesquisa. As comorbidades mais frequentes foram as doenças crônicas como Hipertensão Arterial Sistêmica (HAS) e Hipotireoidismo, altamente prevalentes em idosos, que foi a faixa etária observada. De acordo com o IBGE ${ }^{8}, 75,5 \%$ dos idosos no Brasil são portadores de alguma doença crônica. Em um estudo recente realizado por Soares et al. ${ }^{9}$ em mulheres idosas, foi evidenciado que mais de $98 \%$ da amostra investigada apresentavam ao menos uma morbidade. Tal fato pode aumentar o tempo de internação desses idosos e, consequentemente, os custos com a internação.

No que concerne à modalidade e ao acesso às vias aéreas todos os 14 sujeitos observados estavam sob ventilação mecânica prolongada e ventilavam através de traqueostomia no modo ventilatório de Pressão Positiva Contínua nas Vias Aéreas (CPAP) antes da retirada da prótese ventilatória, sem uso de sedativos, o que permitiu a adequada avaliação do nível de consciência.

Todos os sujeitos estavam em processo de desmame ventilatório seguindo o protocolo institucional de progressão do desmame; portanto, eram retirados da prótese ventilatória diariamente, por períodos pré-programados, de maneira progressiva. Nos 28 eventos a ocorrência mais tardia de uma característica foi aos 205 minutos. Este intervalo de 205 minutos compreendeu a linha do tempo para a distribuição de todas as características.

A linha do tempo foi definida por marcadores temporais de intervalos. Neste aspecto optou-se por seguir uma lógica clínica de intervalos de 15 minutos. Determinados os marcadores de 15 minutos, definiu-se por ponderar a distribuição quantitativa da população de CD em função deles. Procedeu-se, então, ao cálculo dos percentis, 0 que está disposto na Tabela 1.

Tabela 1 - Representação do tempo de avaliação e o correspondente percentil das características definidoras da Resposta Disfuncional ao Desmame Ventilatório observadas/manifestadas nos 28 eventos analisados

\begin{tabular}{cc}
\hline Tempo & Percentil \\
\hline 15 & P18 \\
30 & P33 \\
45 & P45 \\
60 & P53 \\
75 & P66 \\
90 & P76 \\
105 & P89 \\
120 & P95 \\
135 & P96 \\
150 & P97,1 \\
165 & P97,5 \\
180 & P98,5 \\
195 & P99,7 \\
205 & P100 \\
\hline
\end{tabular}


0 que se pode observar, principalmente, é que, aos 30 minutos de observação, 33\% das características definidoras já tinham sido observadas/manifestadas. Avançando no tempo, no marcador dos 120 minutos o percentil 95 foi o correspondente, indicando a manifestação de 95\% das CD.

Com esses resultados, pode-se inferir que a avaliação feita pelo enfermeiro na fase de interrupção da ventilação mecânica no processo de desmame ventilatório deve iniciar com a maior brevidade à retirada da prótese, visto que os sinais e sintomas podem ser identificados quase que imediatamente. Ainda mais, ao permanecer observando os pacientes por ao menos 15 minutos após a retirada da prótese, seria hipoteticamente possível verificar a ocorrência de $18 \%$ das CD, se fossem mantidas as características da amostra pesquisada.

No que diz respeito ao período de duração dos eventos, a interpretação levou a proposição de dois grupos. Observou-se que estes se apresentaram de maneira diversa e que a não separação em diferentes categorias poderia falsear os achados. Os dois grupos principais de eventos foram:

- Eventos de curta duração - caracterizaram-se pelo retorno à prótese ventilatória em um período de tempo inferior a 30 minutos, com manifestação/observação das CDs em um curto intervalo de tempo e com gravidade suficiente para motivar a equipe de Enfermagem a interromper o processo de retirada da ventilação mecânica;

- Eventos com duração típica - caracterizaram-se pelo retorno à prótese ventilatória em um período de tempo superior a 30 minutos.

Para ordenar temporalmente cada característica definidora na distribuição de eventos, foi realizado o cálculo da mediana do tempo de observação/manifestação de cada característica.

Nos eventos de curta duração, as CDs foram observadas/manifestadas quase que imediatamente à interrupção da ventilação mecânica. Como apresentado na Tabela 2, os primeiros sinais foram observados nos primeiros minutos. São detectáveis por meio da inspeção atenta não apenas do padrão respiratório e dos movimentos da parede torácica, mas também das reações comportamentais do paciente, dizendo respeito ao grau de insegurança, ansiedade e à dificuldade de ventilação espontânea. Tais manifestações são sutis e exigem cuidadosa atenção do enfermeiro.

Tabela 2 - Mediana de tempo de observação/manifestação das Características Definidoras da Resposta Disfuncional ao Desmame do Ventilador nos eventos de curta duração (<30 min)

\begin{tabular}{lc}
\hline Características Definidoras & $\begin{array}{c}\text { Mediana } \\
\text { minutos) }\end{array}$ \\
\hline Olhos arregalados & tempo (em \\
\hline Desconforto ao respirar & 1 \\
\hline Inquietação & 3 \\
\hline $\begin{array}{l}\text { Aumento moderado da frequência cardíaca em relação aos parâmetros basais (<20 } \\
\text { batimentos/min) }\end{array}$ & 4 \\
\hline $\begin{array}{l}\text { Aumento moderado da pressão sanguínea em relação aos parâmetros basais (<20 } \\
\text { mmHg) }\end{array}$ & 5 \\
\hline $\begin{array}{l}\text { Sensação de calor } \\
\text { Agitação }\end{array}$ & 5 \\
\hline $\begin{array}{l}\text { Ruídos adventíios na respiração } \\
\text { Secreções audíveis nas vias aéreas }\end{array}$ & 5,5 \\
\hline $\begin{array}{l}\text { Entrada de ar diminuída à ausculta } \\
\text { Aumento da concentração na respiração }\end{array}$ & 6 \\
\hline Uso moderado da musculatura acessória da respiração & 8 \\
\hline Aumento da pressão sanguínea em relação aos parâmetros basais (>20 mmHg) & 8 \\
\hline Respiração abdominal paradoxal & 12,5 \\
\hline $\begin{array}{l}\text { Apreensão } \\
\text { respirações/min) } \\
\text { bumento da frequência cardíaca em relação aos parâmetros basais (>20 }\end{array}$ & 13 \\
\hline $\begin{array}{l}\text { A frequência respiratória aumenta de forma significativa em relação aos parâmetros } \\
\text { basais }\end{array}$ & 10 \\
\hline Respiração superficial & 10 \\
\hline
\end{tabular}


Tabela 3 - Mediana do tempo de observação/manifestação das Características Definidoras da Resposta Disfuncional ao Desmame Ventilatório nos eventos típicos

\begin{tabular}{|c|c|c|}
\hline Características Definidoras & $\begin{array}{l}\text { Mediana do } \\
\text { minutos) }\end{array}$ & tempo \\
\hline Perguntas sobre possível funcionamento inadequado do aparelho & & 19,0 \\
\hline Aumento da concentração na respiração & & 20,0 \\
\hline Sensação de calor & & 25,0 \\
\hline Incapacidade de responder às solicitações verbais & & 32,0 \\
\hline Olhos arregalados & & 35,0 \\
\hline Hipervigilância das atividades & & 36,0 \\
\hline Uso total da musculatura acessória da respiração & & 36,5 \\
\hline Leve diaforese & & 37,5 \\
\hline Aumento moderado da frequência respiratória em relação à linha de base & & 39,0 \\
\hline $\begin{array}{l}\text { Aumento da frequência respiratória em relação aos parâmetros basais }(5< \\
\text { respirações/min) }\end{array}$ & & 40,0 \\
\hline Inquietação & & 40,5 \\
\hline Entrada de ar diminuída à ausculta & & 50,0 \\
\hline Aumento moderado da frequência cardíaca em relação aos parâmetros basais & & 55,0 \\
\hline Uso moderado da musculatura acessória da respiração & & 60,0 \\
\hline Apreensão & & 63,0 \\
\hline Secreções audíveis nas vias aéreas & & 65,0 \\
\hline Ruídos adventícios na respiração & & 70,0 \\
\hline Desconforto ao respirar & & 70,0 \\
\hline Respiração superficial & & 70,5 \\
\hline Agitação & & 72,5 \\
\hline Aumento moderado da pressão sanguínea em relação aos parâmetros basais & & 72,5 \\
\hline $\begin{array}{l}\text { Aumento da frequência cardíaca em relação aos parâmetros basais (> २० } \\
\text { batimentos/min) }\end{array}$ & & 74,0 \\
\hline Respiração ofegante & & 77,0 \\
\hline A frequência respiratória aumenta de forma significativa em relação aos & & 90,0 \\
\hline Aumento da pressão sanguínea em relação aos parâmetros basais (>20 mmHg) & & 95,0 \\
\hline Deterioração nos gases sanguíneos arteriais em relação aos parâmetros basais & & 99,0 \\
\hline Sensação expressa de necessidade de oxigênio aumentada & & 110,5 \\
\hline Fadiga & & 198,0 \\
\hline
\end{tabular}

Já nos eventos típicos (duração superior a 30 minutos) foi possível observar algumas diferenças. De acordo com a ordenação das medianas do tempo de observação/manifestação das Características Definidoras nos eventos típicos (Tabela 3), a primeira característica apresentada tem mediana do tempo de aparecimento de 19 minutos.

Entretanto, não é possível afirmar que os eventos típicos não apresentarão $\mathrm{CD}$ nesse intervalo de tempo, pode-se dizer apenas que, quando nos primeiros 15 minutos de interrupção da VM são observados vários sinais e sintomas sugestivos de RDDV, provavelmente, o desfecho é a necessidade de retorno à prótese ventilatória em menos de 30 minutos.

Em ambos os grupos de eventos, as primeiras características observadas foram manifestações consideradas subjetivas, relacionadas ao comportamento do paciente em relação à interrupção da VM, como olhos arregaladose aumento da concentração na respiração, ou ainda, as relacionadas aos sintomas, como sensação de calor que só podem ser conhecidas por intermédio da observação direta ou da comunicação efetiva com o paciente.
Nas Tabelas 2 e 3 também é possível notar que as CD manifestadas nos minutos finais dos eventos (curtos ou típicos) são as graves de acordo com a classificação da NANDA-I, como $A$ frequência aumenta de forma significativa em relação aos parâmetros basais, Deterioração nos gases sanguíneos arteriais em relação aos parâmetros basaise Respiração superficial.

Os resultados que indicam a manifestação/observação tardia dos sinais de maior gravidade tem bastante coerência clínica. Obviamente, espera-se que sinais e sintomas mais graves ocorram mais frequentemente quanto mais avançado estiver o progresso de instalação da RDDV.

Entretanto, uma CD chamada leve na taxonomia da NANDA-I também foi observada na última etapa dos eventos típicos, a Fadiga. Porém, a gravidade dessa característica, se considerado o quanto compromete a capacidade ventilatória, pode ser questionada.

0 termo Fadiga, de acordo com Mota, Cruz e Pimenta ${ }^{10}$, tem como principais atributos para a sua definição: "cansaço, exaustão, desgaste, alteração da capacidade funcional e falta de recursos/energia". Sendo assim, a fadiga que caracteriza a 
RDDV demonstra o quão difícil estaria a manutenção da ventilação espontânea, critério de gravidade, diferente da classificação da NANDA-I.

Outra questão a ser abordada quando se observam as Tabelas 2 e 3 é que a ordenação cronológica, delimitada por meio das medianas, construiu também uma ordenação fisiopatológica da Resposta Disfuncional ao Desmame Ventilatório, ou seja, a descrição do desenvolvimento fisiológico dos sinais e sintomas que caracterizam a RDDV de forma coerente com a prática clínica.

A partir desses dados, a questão da temporalidade das CD da RDDV começa a ser esclarecida, já que as CD não foram observadas/manifestadas de forma simultânea. Mas, ao contrário, puderam ser observadas/manifestadas em qualquer intervalo de tempo e em tempos diferentes de um mesmo evento, formando uma sequência de sinais e sintomas. Além desse fato, há que se pensar que a maioria das $\mathrm{CD}$ assumiu uma predominância de distribuição no tempo.

Para a determinação da faixa de tempo que representasse as situações de aparecimento precoce, intermediárioe tardioforam determinados os quartis do tempo do evento de duração máxima dos 23 eventos típicos, que foi de 205 minutos.

0 primeiro quartil representando os primeiros $25 \%$ do tempo total da distribuição observado foi considerado como precoce, que caracterizou os primeiros 30 minutos de interrupção da ventilação mecânica (Tabela 4). E o período acima do percentil 75, após o terceiro quartil, foi considerado como tardio, ou seja, as CDs que foram observadas/manifestadas acima dos 90 minutos foram consideradas tardias. Da mesma forma que o chamado intervalo interquartil (equivalente ao segundo e terceiro quartis), o período entre 30 e 90 minutos foi considerado o período intermediário.

Tabela 4 - Distribuição em quartis do tempo de distribuição total das Características Definidoras da Resposta Disfuncional ao Desmame Ventilatório

\begin{tabular}{cc}
\hline Quartis & Tempo (em minutos) \\
\hline $1^{\circ}$ Quartil & 30 \\
$2^{\circ}$ Quartil & 63 \\
$3^{\circ}$ Quartil & 90 \\
Valor Máximo & 205 \\
\hline
\end{tabular}

Assim, a partir da mediana dos tempos em que foi observada cada característica definidora foi possível determinar em qual intervalo de tempo a $C D$ se manifestou predominantemente.

Para melhor visualização, as CD foram distribuídas no Quadro 1 de acordo com a sua predominância.

Quadro 1 - Distribuição das Características Definidoras em relação ao período temporal predominante

\begin{tabular}{|c|c|}
\hline Fenós Toupors' & 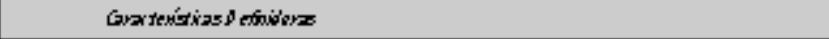 \\
\hline Precate & 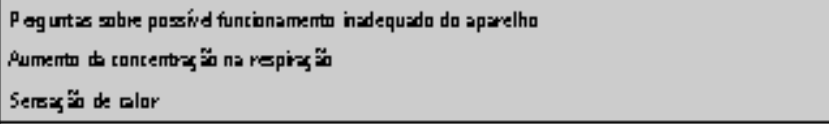 \\
\hline htemedárí & 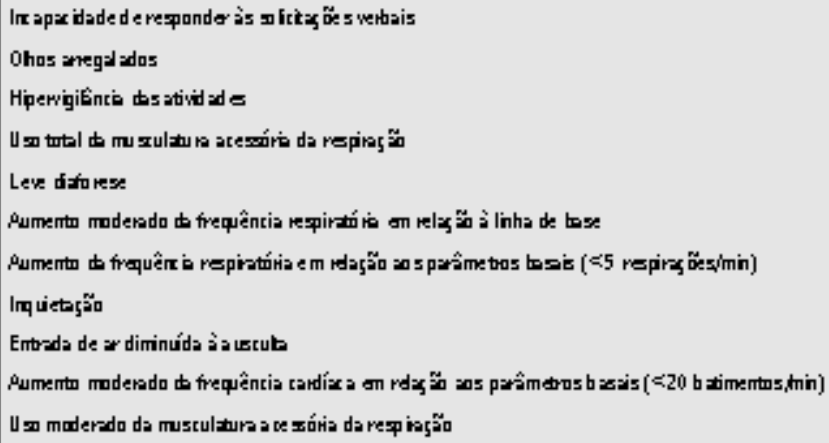 \\
\hline Tardia & 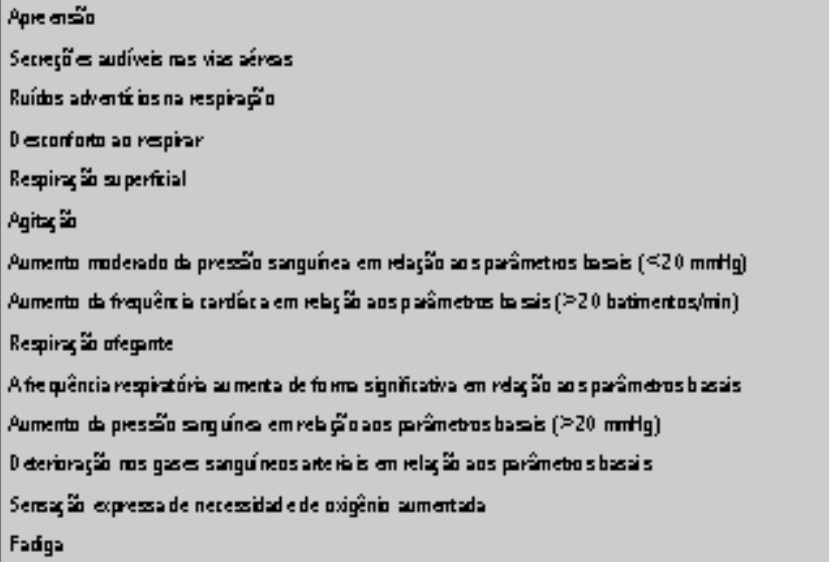 \\
\hline
\end{tabular}


As CDs consideradas precoces, observadas nos primeiros 30 minutos de interrupção da VM foram: "Perguntas sobre possível funcionamento inadequado do aparelho"; "Aumento da concentração na respiração"; e "Sensação de calor". Essas são características que não podem ser detectadas por meio do uso de um monitor multiparâmetros ou da verificação de dados mensuráveis. São características inespecíficas e subjetivas que necessitam do olhar atento da equipe de Enfermagem para que possam ser percebidas, como já discutido anteriormente.

No período intermediário, observa-se o aumento da gravidade das $\mathrm{CD}$ consideradas comportamentais, classificadas como moderadas pela taxonomia da NANDA-I, como Incapacidade de responder às solicitações verbais, Olhos arregalados, Hipervigilância das atividades e Inquietação, corroborando a ideia de aumento da gravidade de acordo com a evolução temporal do fenômeno. Essas características representam o agravamento gradual do quadro na linha do tempo, caracterizando o período intermediário que conecta a fase de alerta à fase de desfecho.

Encontram-se no período tardio, além das características comportamentais, sinais fisiológicos de agravamento do quadro de RDDV, como Secreções audíveis nas vias aéreas, Ruídos adventícios na respiração, Respiração superficial, A frequência respiratória aumenta de forma significativa em relação aos parâmetros basais, Deterioração nos gases sanguíneos arteriais em relação aos parâmetros basais, entre outros descritos no Quadro 1. Dessa forma, é possível supor que outras CD de menor gravidade se manifestaram anteriormente, mesmo que não observadas pela equipe de Enfermagem, ou ainda, se detectadas, não foram valorizadas o suficiente para determinar uma conduta.

Percebe-se que as características se apresentavam de forma distinta, algumas foram precoces, como sinais de alerta para a instalação de uma possível resposta disfuncional, outras foram tipicamente tardias e demonstram a gravidade do quadro e, portanto, motivam o desfecho (o retorno à prótese ventilatória).

Assim, observa-se que o conjunto de características precoces foi formado por sinais subjetivos inespecíficos leves e por sintomas leves. As características intermediárias foram sinais subjetivos moderados e alteração hemodinâmica leve a moderada. Já as características tardias se apresentaram como sinais subjetivos moderados a graves e alteração hemodinâmica moderada a grave.

\section{CONCLUSÔES}

Os achados deste estudo confirmam a existência de padrões temporais das características definidoras da Resposta Disfuncional ao Desmame Ventilatório nos eventos observados. As características não ocorreram de forma simultânea, elas se distribuíram na linha do tempo.

As características definidoras manifestaram-se, principalmente, nas primeiras duas horas; foram observadas quase que imediatamente à retirada da prótese ventilatória; e em 30 minutos mais de $30 \%$ delas já tinham se manifestado. Tais achados destacam a necessidade de o enfermeiro dirigir sua atenção nos primeiros minutos da interrupção da ventilação para identificar os primeiros sinais da RDDV.

Concluiu-se também pela capacidade de identificar padrões predominantes que guardam relação com variáveis temporais. Como visto, a análise dos achados permitiu o reconhecimento de dois tipos predominantes de eventos de Resposta Disfuncional do Desmame Ventilatório (RDDV), a saber: curto e típico. Tais tipos diferenciam a RDDV com prováveis impactos no processo diagnóstico.

Também foi possível distinguir os principais intervalos de observação/manifestação das CD, a saber: precoce, intermediário e tardio, nos quais foram alocadas as características de acordo com as medianas do tempo de observação/manifestação.

A modalidade da pesquisa aqui desenvolvida em um método original nos estudos diagnósticos de enfermagem permitiu que se pensasse na organização do fenômeno diagnóstico como algo passível de um entendimento funcional, no qual a variável de interesse foi o tempo.

No que diz respeito ao gerenciamento do processo de desmame na fase de interrupção da ventilação mecânica, apesar de distribuídas em quase todo intervalo, as CD apresentaram maior percentual de manifestação em determinado período de observação, considerando-se os recortes de 15 minutos estabelecidos, onde o enfermeiro precisaria concentrar sua atenção durante esse processo.

A partir disso, é possível indicar algumas contribuições ao aprimoramento das avaliações protocolares:

(a) a determinação, no protocolo, de intervalos de observação necessários para reduzir a perda de dados relevantes e para otimizar o tempo demandado da equipe de Enfermagem;

(b) a determinação, no protocolo, de necessidade de observação direta pelo enfermeiro nos primeiros 15 minutos de interrupção da VM, entendido como determinante para aparecimento de CD e para a evolução da RDDV;

(c) incluir no programa de treinamento da equipe de Enfermagem a descrição das CD do diagnóstico de RDDV, levando em consideração a variável tempo; (d) inserir no prontuário eletrônico da instituição a RDDV, com suas respectivas características definidoras e fatores relacionados, para melhorar a avaliação e implantação de medidas cabíveis de intervenção.

É fato que outros estudos devam ser realizados em condições controladas para complementação, confrontamento e/ou para corroborar os resultados discutidos na pesquisa em tela. Assim, sugere-se a realização de estudos com amostras diferentes da observada nessa pesquisa, como pacientes jovens, com outras comorbidades, e que sejam utilizados outros modelos de protocolo de desmame, para verificar se existe similaridade com os resultados apontados nesta pesquisa. 
Também se propõe a reprodução desse estudo com um número maior de eventos e, de preferência, estudos multicêntricos com o objetivo de confrontar os dados apresentados até o momento.

\section{REFERÊNCIAS}

1.Brandão MAG. Uma visão metacognitiva das estratégias diagnósticas de enfermagem. [dissertação]. Rio de Janeiro: NUTES/UFRJ; 1999.

2.Carnevali DL, Thomas MD. Diagnostic reasoning and treatment decision making in nursing. Philadelphia: J.B. Lippincott Company; 1993.

3.Levin RF, Lunney M, Krainovich-Miller B. Improving diagnostic accuracy using an evidence-based nursing model. Inter I Nurs Terminol Classif. 2004; 15(4): 114-22.

4.Sousa VD, Driessnack M, Mendes IAC. An overview of research designs relevant to nursing: part 1: quantitative research designs. Rev Latino-Am Enfermagem. 2007; 15(3): 502-07.

5. $3^{\circ}$ Consenso Brasileiro de Ventilação Mecânica. J Bras Pneumol. 2007; 33(supl 2):S51-S150.

6.Ministério da Saúde(BR). Departamento de Informática do SUS- DATASUS. Morbidade hospitalar do SUS. Brasil; 2010.

7.Constantino SS. Desmame ventilatório: Índices preditivos e prognóstico de pacientes em ventilação mecânica invasiva [dissertação] Rio de Janeiro: Faculdade de Medicina, Universidade Federal do Rio deJaneiro; 2007.

8.Instituto Brasileiro de Geografia E Estatística- IBGE. Indicadores sociodemográficos e de saúde do Brasil 2009. Rio de Janeiro; 2009.

9.Soares MBO, Tavares DMS, Dias FA, Diniz MA, Geib S. Morbidades, capacidade funcional e qualidade de vida de mulhereres idosas. Esc Anna Nery. 2010; 14 (4): 705-11.

10.Mota DDCF, Cruz DALM, Pimenta CAM. Fadiga: uma análise de conceito. Acta Paul Enferm. 2005; 18(3): 285-93. 